\title{
Regional Contributions to Isentropic Pressure Torques
}

\author{
JOSEPH EGGER \\ Meteorologisches Institut, Universität München, Munich, Germany \\ KLAus-Peter HoinKa \\ Institut für Physik der Atmosphäre, DLR, Oberpfaffenhofen, Germany
}

(Manuscript received 18 August 2009, in final form 1 April 2010)

\begin{abstract}
Transfer of axial angular momentum across isentropic surfaces due to adiabatic processes is performed by pressure torques. These torques are evaluated from observations for selected latitude belts and isentropic surfaces, focusing attention on regional contributions. It is found that downward time mean contributions culminate in the storm tracks except above and near major mountain massifs where even upward transfers may be found. Variations of these torques in time are short lived with a decay time of 1-2 days. Height perturbations of isentropic surfaces are presented for torque events. The torque patterns are compared to analyses of the more conventional vertical momentum transports in the $z$ system.
\end{abstract}

\section{Introduction}

Our view of the axial angular momentum (AAM) balance in the global atmospheric circulation depends crucially on the choice of the coordinate system for the analysis. For example, the zonal and time mean flow in the meridional plane is dominated by the tropical Hadley cell (e.g., Oort and Peixoto 1983) in the $z$ system, while there is little mean meridional circulation outside the tropics. The strongest fluxes of AAM are restricted to the tropical belt. On the other hand, there is a direct cell spanning the whole winter hemisphere in isentropic coordinates (e.g., Johnson 1989), which transports AAM from the equator to the Pole. As pointed out by Andrews (1983), Johnson (1989), and others, there are large pressure torques that affect the AAM balance in isentropic coordinates. They are, however, not found in AAM budgets in $z$ coordinates. This "discrepancy" is resolved easily by recalling that AAM budgets are normally performed with respect to zonal annuli of constant meridional width $a D \varphi$ and surfaces of constant height or potential temperature $\theta$ as upper boundaries. The pressure torque exerted at such a surface is

Corresponding author address: Joseph Egger, Meteorological Institute, University of Munich, Theresienstr. 37, 80333 Munich, Germany.

E-mail: j.egger@lrz.uni-muenchen.de

$$
T_{o}=\int_{\varphi_{1}}^{\varphi_{2}} \int_{0}^{2 \pi} p \frac{\partial h}{\partial \lambda} a^{2} \cos \varphi d \lambda d \varphi
$$

where $h$ is the height of the coordinate surface and $p$ the related pressure $\left[D \varphi=\varphi_{2}-\varphi_{1}\right.$; see also (1.8)]. Obviously $T_{o}=0$ if $h$ is constant as in the $z$ system. On the other hand, $T_{o}>0$ if the pressure tends to be anomalously large (small) in upslope (downslope) regions. Pressure torques with respect to the annuli are possible in isentropic AAM budgets because $\theta$ surfaces are of variable height. The same torques would be found in the $z$ system if one would consider the same volumes as in the $\theta$ system.

Of course, (1.1) is also correct if $h$ is the topography and $p$ is the surface pressure so that $T_{o}$ is the mountain torque (except for the sign). While the position and shape of the mountains is of obvious regional importance for the mountain torques, the situation with respect to the $\theta$ system is more complicated because the height of a $\theta$ surface changes in time. It is not clear a priori where the largest contributions to the pressure torque will be found. They are presumably strong in areas with large variability of pressure and the height of $\theta$ surfaces. One may speculate that contributions are large in the storm tracks, but, to the best of our knowledge, the related problems have not been investigated yet. Which areas contribute prominently to the pressure torques? Which time scales are involved? Given the importance of the pressure torques in the AAM budget 
(Johnson 1989), it appears necessary to deal with these questions if we want to understand the isentropic AAM budget. It is the purpose of this paper to investigate the regional contributions to $T_{o}$ on the basis of observations.

Although (1.1) gives the correct pressure torque it is necessary to rederive (1.1) so as to better see regional effects. Let us consider the angular momentum equation in $\theta$ coordinates:

$$
\frac{\partial}{\partial t} J m+\nabla_{2} \cdot\left(J m \mathbf{v}_{2}\right)+\frac{\partial}{\partial \theta}(J m \dot{\theta})=-J \frac{\partial M}{\partial \lambda},
$$

where frictional terms are omitted,

$$
m=(u+\Omega a \cos \varphi) a \cos \varphi
$$

is specific AAM,

$$
J=-g^{-1} \frac{\partial p}{\partial \theta}
$$

is "density," and $\dot{\theta}$ is the diabatic heating. The Montgomery potential is

$$
M=c_{p} T+g h
$$

with height $h$ of the isentropes. Its derivative in (1.2) captures the zonal acceleration. The Montgomery term is rewritten as

$$
\begin{aligned}
\frac{\partial p}{\partial \theta} \frac{\partial M}{\partial \lambda}= & \frac{\partial}{\partial \theta}\left(p \frac{\partial M}{\partial \lambda}\right) \\
& -c_{p} R\left(c_{p}+R\right)^{-1} p_{\mathrm{oo}}^{-R / c_{p}} \frac{\partial}{\partial \lambda}\left[p^{\left(R+c_{p}\right) / c_{p}}\right],
\end{aligned}
$$

where we exploit the relation:

$$
\frac{\partial M}{\partial \theta}=c_{p}\left(\frac{p}{p_{\mathrm{oo}}}\right)^{R / c_{p}}
$$

with reference pressure $p_{\mathrm{oo}}$. The relation in (1.6) is a special case of the general equation (44) of Johnson (1980). The contribution by adiabatic processes is represented by the last term on the left-hand side of (1.2). Heating enhances the potential temperature of the air. An air parcel "moves upward" in $\theta$ coordinates if $\dot{\theta}>0$ and so does the related AAM.

AAM budgets require integrations of (1.2) over annuli in $\theta$ space as discussed above. Let us consider again a zonal annulus of "width" $\varphi_{1}<\varphi<\varphi_{2}$ with a lower (upper) boundary isentropic surface $\theta=\theta_{1}\left(\theta=\theta_{2}\right)$. Zonal, vertical, and meridional integration of (1.1) yields the torque difference:
$T_{o}\left(\theta_{2}\right)-T_{o}\left(\theta_{1}\right)=\left.\int_{\varphi_{1}}^{\varphi_{2}} \int_{0}^{2 \pi}\left(p \frac{\partial h}{\partial \lambda}\right)\right|_{\theta_{1}} ^{\theta_{2}} a^{2} \cos \varphi d \lambda d \varphi$

with respect to the annulus where $T_{o}\left(\theta_{i}\right)$ is the pressure torque at the surface $\theta_{i}$. A positive torque implies downward transfer. It has been assumed tacitly in (1.8) that the isentrope $\theta=\theta_{1}$ does not intersect the surface of the earth. The torque $T_{o}\left(\theta_{i}\right)$ describes the transfer of AAM across the $\theta$ surface due to pressure stresses (e.g., Johnson 1980). This term is a torque as becomes obvious by the fact stated above that $T_{o}$ has the same form and physical meaning as the mountain torque. Note that Johnson $(1980,1989)$ calls the vertical derivative of $T_{o}$ a torque. The contribution of the temperature term $c_{p} T$ to $M$ drops out in (1.8) because temperature is a function of pressure on an isentropic surface. The same way, the second term on the right-hand side of (1.6) does not contribute to the pressure torque. On the other hand, both terms would have to be included if we were to evaluate AAM budgets of regional volumes.

Although we will concentrate on pressure torques a comment on the role of diabatic heating is appropriate. The related fluxes of angular momentum are quite important. In particular, the part $m_{m}=\Omega a^{2} \cos ^{2} \varphi$ of specific AAM, the so-called mass term, can be transferred across isentropes only by heating. The related prognostic equation is

$\frac{\partial}{\partial t} J m_{m}+\nabla_{2} \cdot\left(J m_{m} \mathbf{v}_{2}\right)+\frac{\partial}{\partial \theta}\left(J m_{m} \dot{\theta}\right)=-J v 2 \Omega a \cos \varphi \sin \varphi$,

which does not contain the pressure torques. These torques transfer only the so-called wind term $m_{w}=$ ua $\cos \varphi$. After all, $\theta$ is conserved in adiabatic processes so that no mass is exchanged between layers.

While the torques represent integrals over a ring's surface we are able to single out regional contributions by investigating the variations of the integrand $p \partial h / \partial \lambda$ with longitude, latitude, and time. This way we extract the desired information from the data. Where does the main vertical transfer occur and what are the related time scales? We stress again, however, that this term correctly describes the regional contribution to the zonal mean but not the total regional transfer.

We may introduce with

$$
v_{g}=(f a \cos \varphi)^{-1} \frac{\partial M}{\partial \lambda}
$$

the meridional geostrophic wind and replace the pressure by the temperature in (1.8) using 


$$
p=p_{\mathrm{oo}}(T / \theta)^{c_{p} / R}
$$

to find

$$
T_{o}\left(\theta_{i}\right)=\int_{\varphi_{1}}^{\varphi_{2}} \int_{o}^{2 \pi} p_{\mathrm{oo}}\left(T / \theta_{i}\right)^{c_{p} / R} v_{g} f a^{2} \cos ^{2} \varphi d \lambda d \varphi
$$

where $f=2 \Omega \sin \varphi$ is the Coriolis parameter. The torques are obviously related to the meridional temperature transport by the geostrophic wind. Similar relations have been proposed by Juckes et al. (1994) and Tanaka et al. (2004).

The relation in (1.12) demonstrates the close connection of the torque $T_{o}$ and the standard formulation for the vertical component of the Eliassen-Palm flux (e.g., Andrews 1983; Edmon et al. 1980). Similarly, Johnson and Downey (1975), Townsend and Johnson (1985), Johnson (1989), and others argued that the structure of baroclinic systems implies positive pressure torques. As a matter of fact, a look at the structure of unstable Eady waves (e.g., Fig. 8.10 of Holton 1992) tells us almost immediately that such waves would exert a downward pressure torque. Pressure maxima are located west of the corresponding isentropic height extrema so that $T_{o}>0$. Altogether we expect that regional contributions to the pressure torque culminate in the storm tracks where sensible heat transports are largest. We have to stress, however, that (1.12) contains the contribution of the temperature term $c_{p} T$ to the Montgomery potential while (1.8) does not. Moreover, the integrands of (1.8) and (1.12) are not identical at a local level.

The relation of mountain and pressure torques has been investigated by Egger and Hoinka (2009) who correlated the mountain torque and isentropic pressure torques $T_{o}\left(\theta_{i}\right)$ for various latitude belts and isentropic surfaces. One might expect that pressure torques transfer that AAM downward, which is removed at the mountains. The analysis of Egger and Hoinka (2009) supports this view for the polar belts and also for a northern midlatitude belt. The relation of the torques is, however, quite complicated in the subtropics. No simple model is available to explain these findings.

\section{Data and methods}

This work is based on data of the European Centre for Medium-Range Weather Forecasts (ECMWF) ReAnalysis (ERA) for the years 1958-2001. Standard procedures provide the height and pressure of selected $\theta$ surfaces and the related pressure on them. The $\theta$ surfaces with $\theta=295,300,310,320,330,350$, and $370 \mathrm{~K}$ have been selected for this investigation. They represent the troposphere and the lower stratosphere. Some of them intersect normally the ground. Five wide belts are considered for budget calculations: Two of them cover the polar caps (belts PS: $90^{\circ} \mathrm{S}<\varphi<72^{\circ} \mathrm{S}$ and PN: $72^{\circ} \mathrm{N}<\varphi<$ $90^{\circ} \mathrm{N}$ ), two represent the midlatitudes (belt MS: $58^{\circ} \mathrm{S}<$ $\left.\varphi<40^{\circ} \mathrm{S} ; \mathrm{MN}: 40^{\circ} \mathrm{N}<\varphi<58^{\circ} \mathrm{N}\right)$, and one covers the tropical belt (belt TE: $9^{\circ} \mathrm{S}<\varphi<9^{\circ} \mathrm{N}$ ). The torques $T_{o}\left(\theta_{i}\right)$ are evaluated once a day for all belts. ERA analyses are not performed in isentropic coordinates. The necessary interpolations to one $\theta$ surface imply a certain loss of accuracy.

Time mean fields are of particular relevance for budgets. The zonal mean distribution of the time mean pressure torques is presented in Egger and Hoinka (2008), but is given again here for the sake of completeness (Fig. 1; see also Fig. 25e of Johnson 1989). The torques are positive outside the tropical belt and parts of the polar caps. Maxima of 20-30 Hadley (1 Hadley $\left.=10^{18} \mathrm{~J}\right)$ for $D \varphi=$ $4.5^{\circ}$ are found in midlatitudes at $\theta \sim 290 \mathrm{~K}$. Note that a torque of 10 Hadley acting on an atmospheric annulus of 1-km depth and $4.5^{\circ}$ width close to the ground corresponds with a zonal acceleration of $\sim 1.6 \times 10^{-4} \mathrm{~m} \mathrm{~s}^{-2}$ for this volume in midlatitudes. The derivative $\partial T_{o} / \partial \theta$ is negative above the maxima and positive below so that the lower troposphere in midlatitudes receives angular momentum, which is transferred downward from the lower stratosphere and upper troposphere. Note that Johnson (1989) presents essentially this vertical derivative. There are hardly any pressure torques in the tropics.

The pressure torques in Fig. 1 are due to atmospheric eddies which deviate from the zonal mean. Stationary as well as transient motions contribute to this vertical transfer. It is of interest to see the role of such motions in the $z$ system. Although an exhaustive intercomparison of AAM budgets in both systems is far beyond the scope of this paper, the zonal mean of the transient flux $\bar{\rho} \overline{u^{\prime} w^{\prime}} a \cos \varphi$ (bar time mean; prime deviation) has been evaluated as well (not shown). Upward flux maxima of 2 Hadley are found at $25^{\circ} \mathrm{N}, z \sim 8 \mathrm{~km}$ and the strongest downward fluxes of approximately -0.5 Hadley occur near $60^{\circ}, z \sim$ $2 \mathrm{~km}$. Thus, the transient fluxes in the $z$ system are quite weak compared to the pressure torques. Their zonal mean distribution deviates strongly from that of the pressure torques. We will comment on these findings below.

As stated above, we are mainly interested in the regional contributions to the zonal means, that is, we have to present the regional torque contribution:

$$
\overline{T_{\text {or }}}=\int_{F} \overline{p^{*} \frac{\partial h}{\partial \lambda}} d F
$$

where the bar stands for a seasonal time mean. It is convenient for the calculation of the regional contributions 


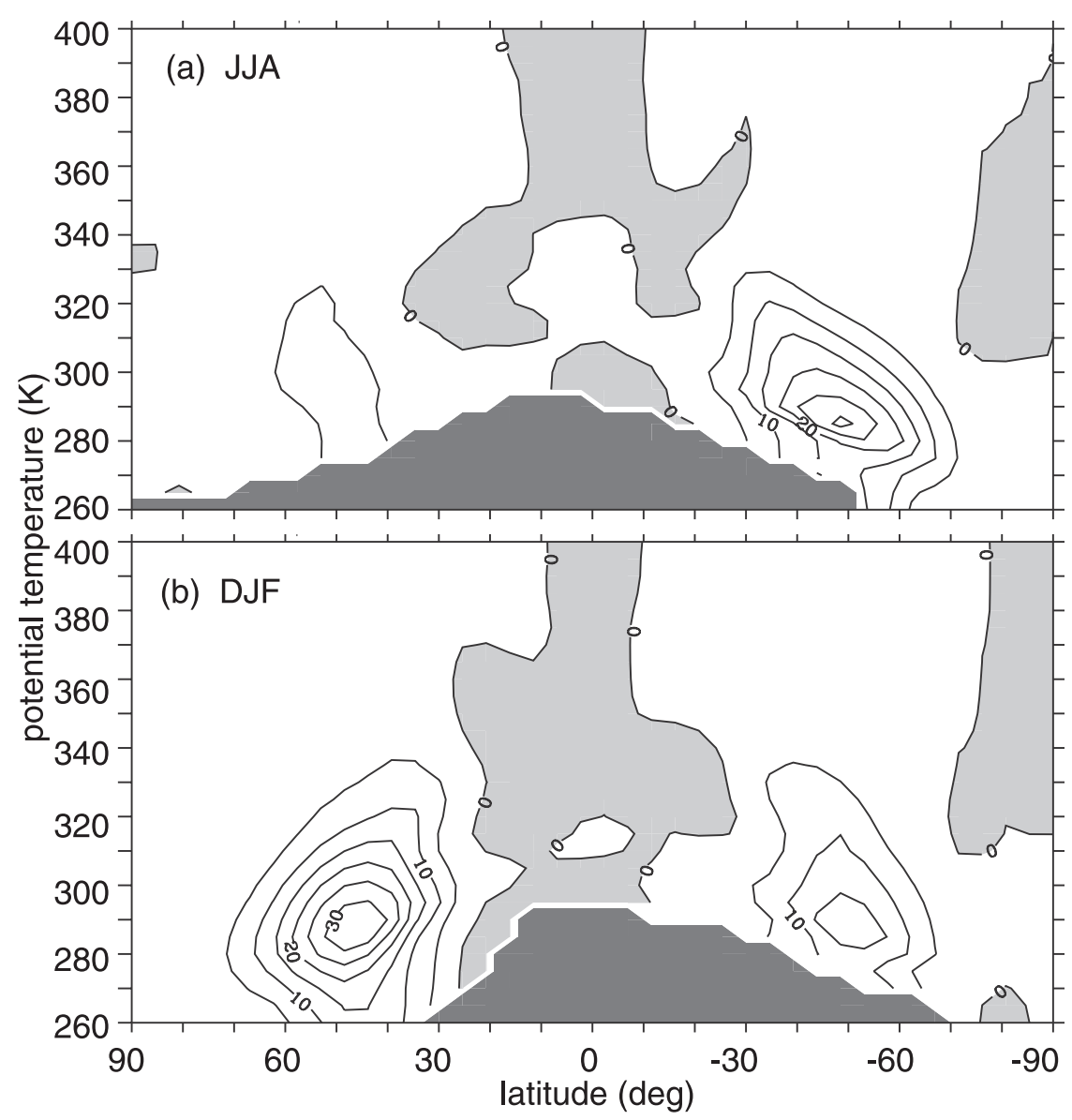

FIG. 1. Isentropic time mean pressure torque $\overline{T_{o}}(\theta)$ in Hadley for (a) JJA and (b) DJF for belts of width $D \varphi=4.5^{\circ}$ as a function of potential temperature and latitude. Adapted from Fig. 1 in Egger and Hoinka (2008). Light shading: negative values; dark: regions below the surface; units: 1 Hadley $=10^{18} \mathrm{~J}$.

to introduce with $p^{*}$ the deviation of the pressure at a certain height from that of a standard atmosphere [see also section $3 \mathrm{a}$ for further comments on the role of $p^{*}$ in (2.1)]. The integration in (2.1) is performed for small $\left(1.125^{\circ} \times 1.125^{\circ}\right)$ rectangles $F$ in a $(\lambda, \varphi)$ grid on an isentropic surface $\left(d F=a^{2} \cos \varphi d \lambda d \varphi\right)$. Thus, (2.1) describes the contribution of this area to the global pressure torque. The pressure of a standard atmosphere would generate in (2.1) large regional torques at the slopes of the stationary waves, which would, however, cancel in the zonal integrations. By introducing $p^{*}$ in (2.1) we do not alter the zonal mean torque. Although (2.1) quantifies the contribution of the area $F$ to the global pressure torque, it is by no means certain that $\overline{T_{\text {or }}}$ is a major term in the AAM balance of a regional air mass extending from the earth's surface up to the $\theta$ surface of (2.1). Pressure torques act at the zonal walls of this volume, which are generally large (e.g., Egger et al. 2007). Zonal convective transfers may be important as well.
The evaluation of the torques $T_{o}(\theta)$ becomes difficult if the $\theta$ surface intersects the topography. Although a correct formulation of the resulting terms is possible, the corresponding evaluation of these orographic corrections from data is not meaningful given the relatively coarse resolution of the dataset. It has been decided to accept the values at the first $\theta$ surface above the ground as the contribution to the total torque. On the other hand, we simply assume $T_{\text {or }}=0$ if most of $d F$ is underground.

Although the presentation of the regional time mean contributions is the central theme of this paper it is also of interest to look at the relation of $T_{\text {or }}$ to the pressure torques of the belts. Does an intensification of the torque of a belt automatically lead to an increase of $T_{\text {or }}$ throughout the belt area? Are there preferred areas with enhanced contributions? Let us denote by $C(b, c \mid \tau)$ the covariance of variable $b$ and $c$ where $b$ is leading with lag $\tau$. Hence, we wish to look at $C\left(T_{o}, T_{\text {or }} \mid \tau\right)$ for selected $\theta$ surfaces. 


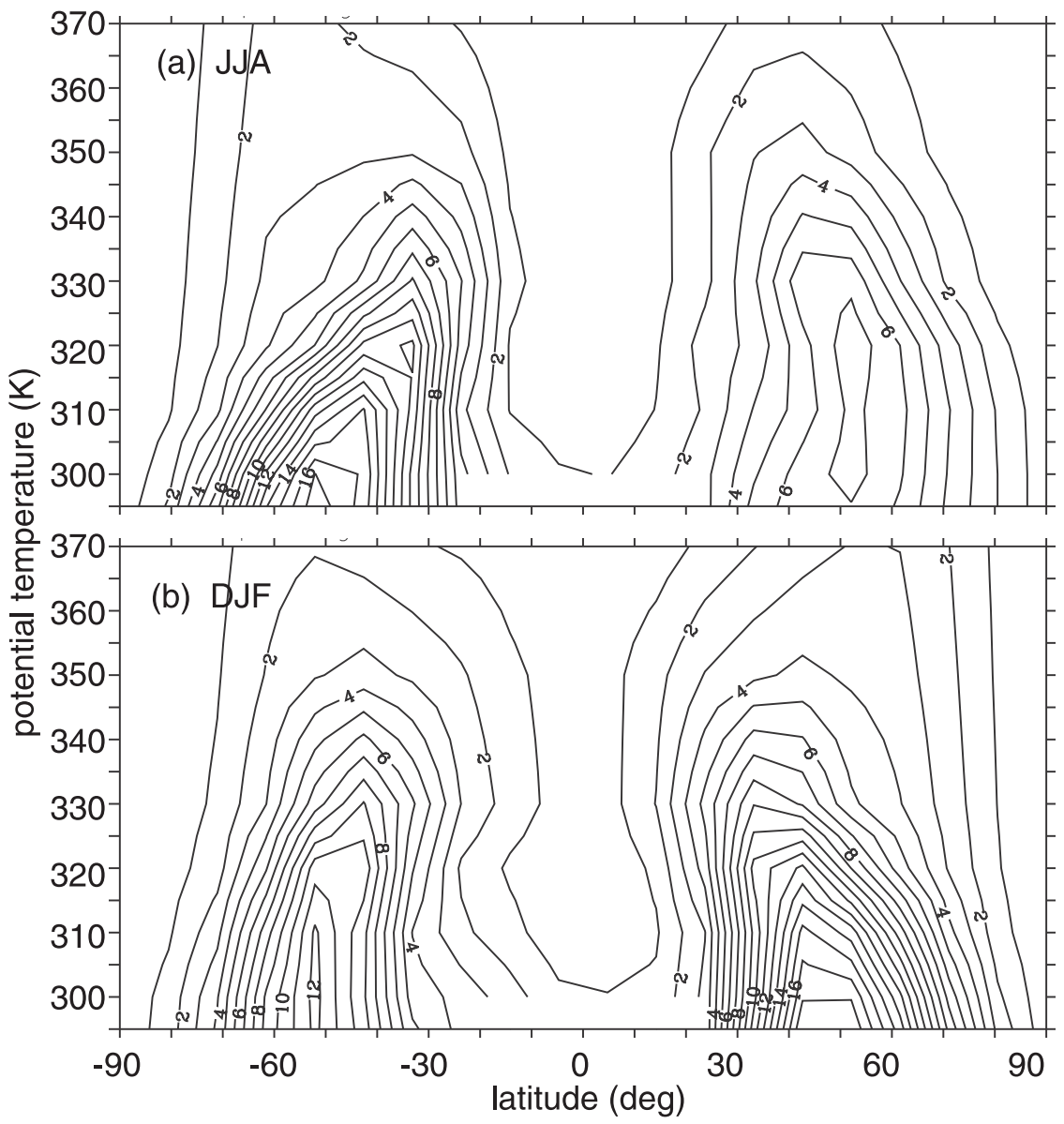

FIG. 2. Standard deviation of the pressure torque per belt of width $D \varphi=4.5^{\circ}$ in Hadley as a function of latitude and potential temperature in (a) JJA and (b) DJF.

\section{Results}

\section{a. Time mean torque statistics}

The standard deviations of the pressure torques per belt of a width $D \varphi=\left(\varphi_{2}-\varphi_{1}\right)=4.5^{\circ}$ are displayed in Fig. 2 for December-February (DJF) and June-August (JJA). Maxima of $\sim 17$ Hadley are found in the respective winter hemisphere at midlatitudes near $\theta=$ $300 \mathrm{~K}$. There is an equatorward tilt of the distributions with increasing height. A similar tilt is seen in presentations of the time mean isentropic Eliassen-Palm flux (Tanaka et al. 2004). As in Fig. 1, the tropical domain is almost free of pressure torques and stratospheric values are quite small. Mean values (Fig. 1) and standard deviations are of the same order of magnitude so that the variability of the torques is substantial in midlatitudes.

The regional time mean contributions $\overline{T_{\text {or }}}$ to the pressure torque are displayed in Fig. 3. There is strong downward tranfer of zonal momentum on the $300-\mathrm{K}$ surface (Fig. 3a) in the storm track regions in the eastern Pacific and over most of the Atlantic in DJF. Negative mean contributions (upward transfer) exist near the west coast of Greenland and above the northwestern Pacific. There is a belt of positive torque contributions in the Southern Hemisphere midlatitudes with a conspicuous minimum above the southern tip of America. Higher up $(\theta=310 \mathrm{~K}$; Fig. 3b), the intersections with the ground are restricted to the major mountain ranges for $|\varphi|<50^{\circ}$. Otherwise, the pattern in DJF is fairly similar to that in Fig. 3a. Amplitudes are less than on the 300-K surface as one would expect (see also Fig. 1). Values above the Northern Cordillera are low when compared to those in the storm tracks in the east and west. The same is true for the Andes Mountains. Note the wide area of negative torques west of the United States. Angular momentum is transferred upward there and west of Spain. Torque contributions in the tropical belt are mainly weak and negative. There is mainly noise over Europe and large parts of Asia. The similarity of Fig. $3 b$ to the 2-6-day high-pass-filtered variances of various variables in Hoskins and Hodges (2002) is conspicious. Negative contributions in Fig. 3 coincide roughly 

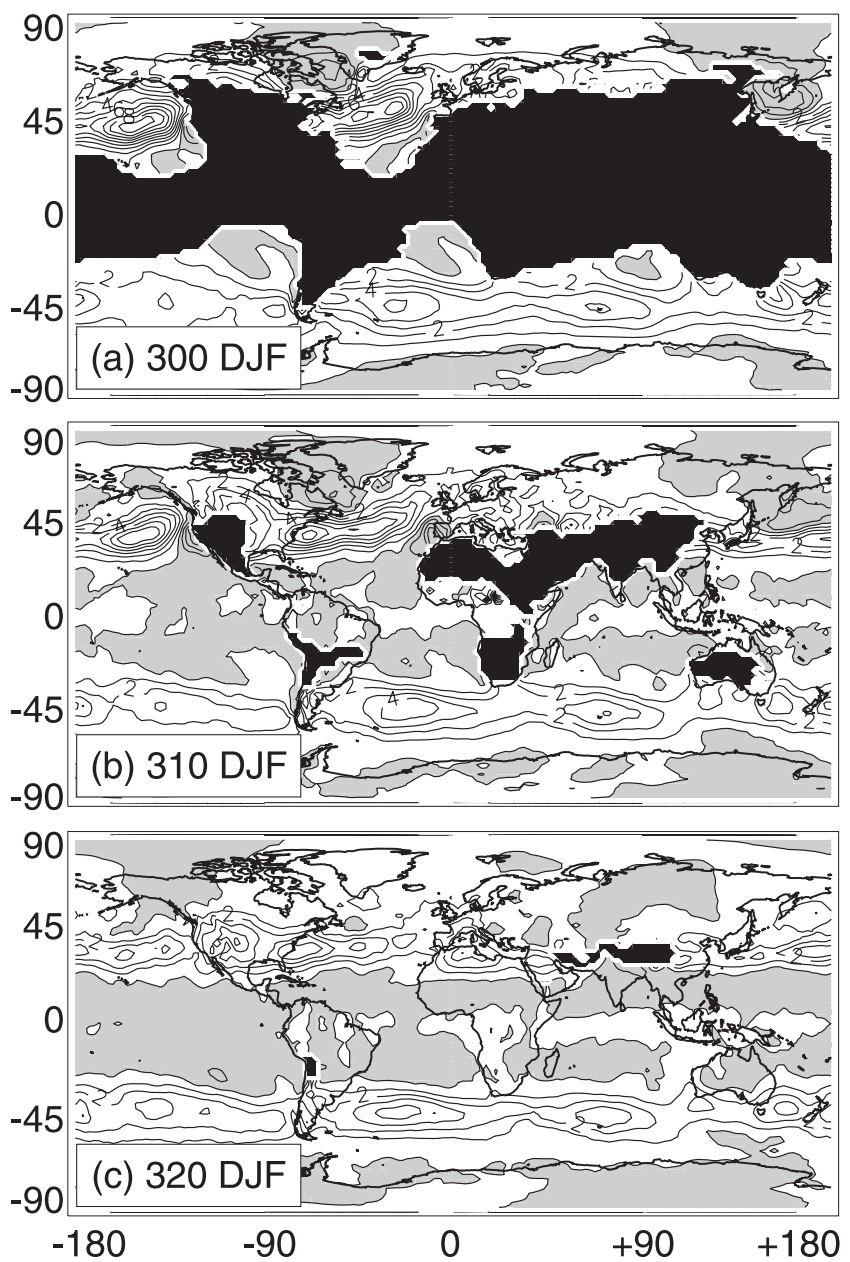
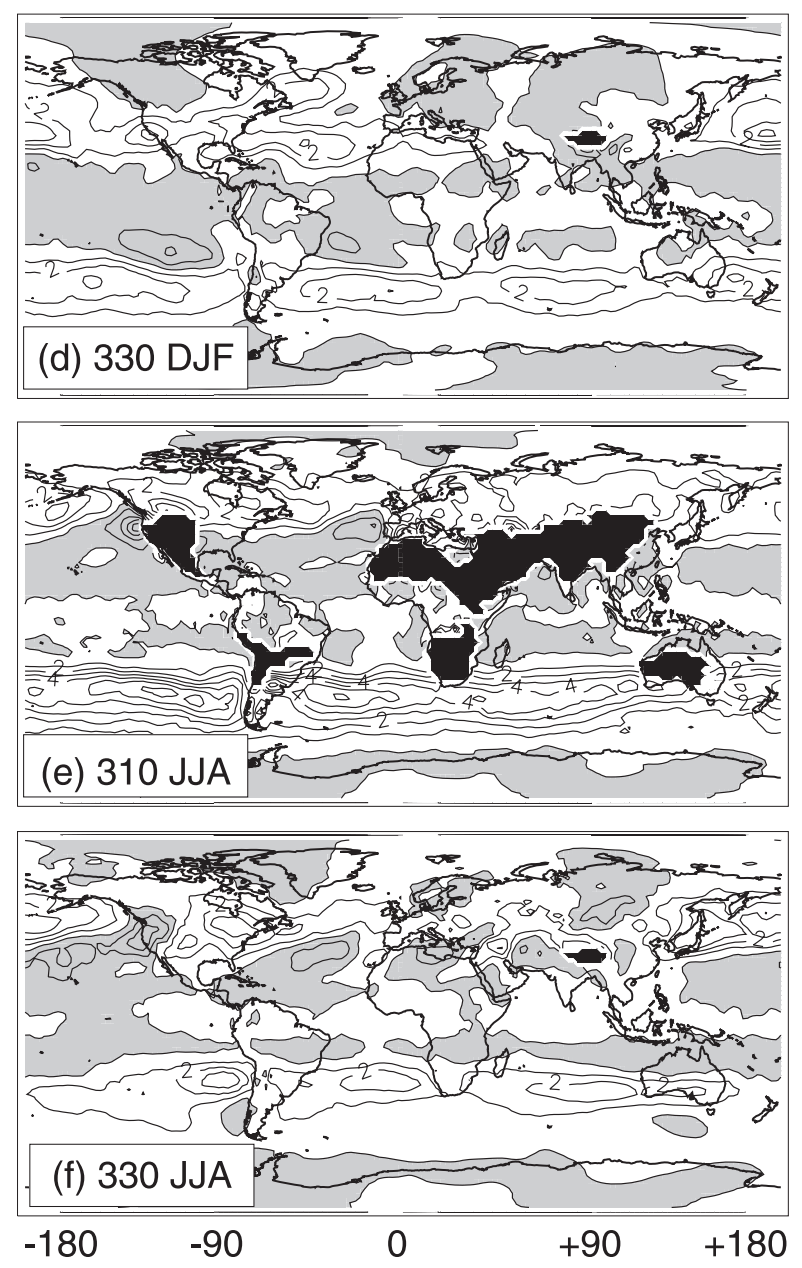

FIG. 3. Time mean regional torque contributions $\bar{T}_{\text {or }}$ for $1.125^{\circ} \times 1.125^{\circ}$ grid "squares" for (a) $\theta_{1}=300 \mathrm{~K}$, DJF; (b) $\theta_{1}=310 \mathrm{~K}$, DJF; (c) $\theta_{1}=320 \mathrm{~K}, \mathrm{DJF}$; (d) $\theta_{1}=330 \mathrm{~K}$, DJF; (e) $\theta_{1}=310 \mathrm{~K}$, JJA; and (f) $\theta_{1}=330 \mathrm{~K}$, JJA. Shading: negative values; dark: the $\theta$ surface is "below" the ground at least on 1 day; contour interval: 0.01 Hadley.

with domains of little activity in these maps, while strong activity is typically found in the areas where $\overline{T_{\text {or }}}$ is large. Domains of little synoptic variability tend to be domains of upward angular momentum transfer. An intercomparison with maps of meridional heat transports (Oort and Peixoto 1983) as suggested by (1.12) shows quite good agreement except that the strong torque contributions in the northeastern Pacific have only weak heat transports as counterpart. There are no negative heat transports east of Spain. At $\theta=320 \mathrm{~K}$ there is a tendency to have relatively strong torque contributions in a fairly narrow "subtropical" belt (Fig. 3c). This corresponds with the southward shift of maxima seen in Fig. 1. Note the conspicuous maxima over the western Mediterranean and North Africa as well as east of the North American Cordillera. Although the Mediterranean is known as a center of activity in winter, it is surprising that the corresponding maximum of $\overline{T_{\text {or }}}$ is not found at $\theta=310 \mathrm{~K}$. There is no minimum above the Northern Cordillera any more. There are only weak positive contributions above northeastern Asia. A split of the Atlantic maximum into two "branches" is seen at $\theta=330 \mathrm{~K}$ (Fig. 3d). The Mediterranean maximum is not represented at $\theta=330 \mathrm{~K}$ and there is now a maximum above California. Amplitudes are quite small higher up but there is a maximum above the northeastern Atlantic and the eastern Pacific even on the 350-K surface (not shown).

In JJA it is, of course, the storm track region in the Southern Hemisphere where the largest values are found with a minimum above the Andes region (Fig. 3e). There is little variation of this pattern if $\theta$ increases from 310 (Fig. 3e) to $330 \mathrm{~K}$ (Fig. 3f).

As argued above it is revealing to compare the regional torque contributions in Fig. 3 to the vertical mean transient transports $\bar{\rho} \overline{u^{\prime} w^{\prime}} a \cos \varphi$ of the wind term in the $z$ system as displayed in Fig. 4 for the midtropospheric 


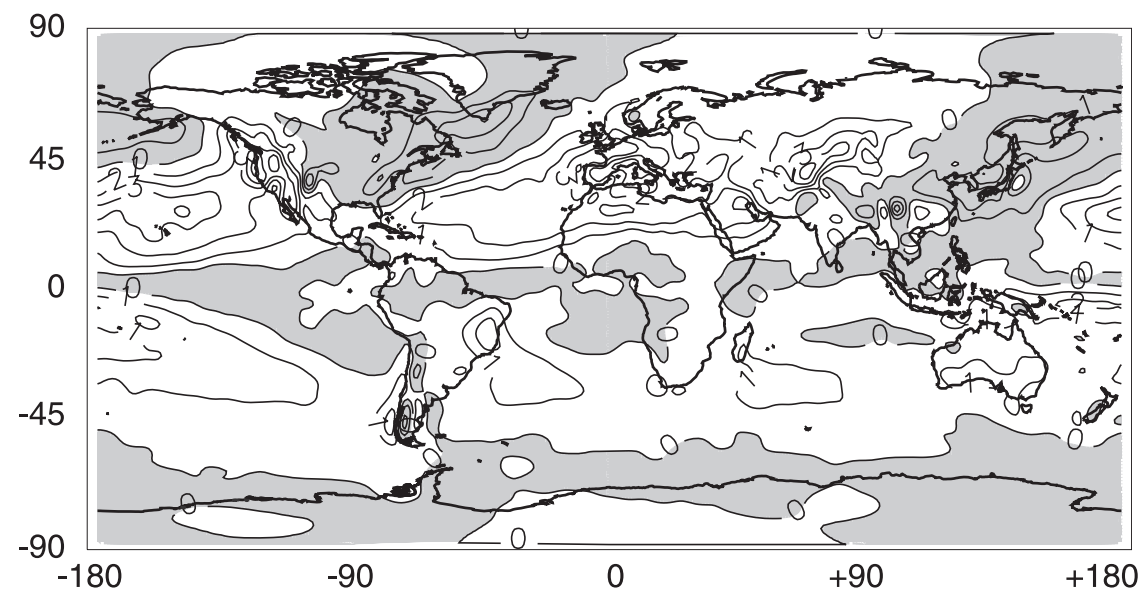

FIG. 4. Time mean vertical flux $\rho_{o} \overline{u^{\prime} w^{\prime}} a \cos \varphi$ of the wind term at the constant height $z=6 \mathrm{~km}$ for $1.125^{\circ} \times 1.125^{\circ}$ grid squares as in Fig. 3, contour interval 0.001 Hadley. The data field is smoothed by applying a nine-point smoother.

height $z=6 \mathrm{~km}$. Of course, the height of isentropic surfaces decreases with decreasing latitude, but the patterns in Fig. 4 do not vary strongly with height so that at least a qualitative intercomparison is feasible. In general, the fluxes in Fig. 4 are small when compared to the corresponding torque contributions in Fig. 3. Wind term tranports are directed upward in the storm tracks of the Southern Hemisphere and of the NH Pacific and Atlantic. Downward transport occurs mainly on the polar side of the storm tracks. By and large, the fluxes in the $z$ system and the torques tend to have opposite directions.

This leads to a puzzling situation. Consider a ring in midlatitudes extending from the surface to a height of $6 \mathrm{~km}$. This ring loses AAM at its upper boundary due to eddy motion. Let us next choose a $\theta$ surface of height close to $6 \mathrm{~km}$ as upper boundary so that both rings contain about the same air mass. This $\theta$ ring gains large amounts of AAM due to the pressure torque. There is loss of AAM due to surface friction at the ground. Thus, AAM must be imported through the lateral boundaries if the upper boundary is of constant height but must be presumably exported if the isentropic surface tops the volume. Thus, the meridional AAM fluxes must differ profoundly. This is possible only because the lateral boundary surfaces of both rings differ, the ones being of constant height while the height of the other ones is that of the $\theta$ surface.

\section{b. Lagged torque covariances}

The time scale of torque events is only a few days as can be seen from Fig. 5 where the autocovariances of the torques $T_{o}$ are displayed for all five belts. Of course, the midlatitude belts MS and MN exhibit the largest variances by far. The decay is rapid with a zero crossing for
$2 \leq \tau \leq 3$ days. The situation is similar for the polar belt PN except that the variance is much smaller and summer is more active. The small variances in the polar belts (see also Fig. 2) reflect, of course, also the reduction of belt area with increasing latitude. The pressure torques reflect the phase relations of height and pressure fields on a $\theta$ surface. The zero crossings in Fig. 5 tell us that favorable constellations decay within a few days.

Let us next look at the covariances $C\left(T_{o}, T_{\mathrm{or}} \tau\right)$ of the regional contributions and the pressure torque of a belt as a function of lag $\tau$ (Fig. 6). These fields tell us which regions provide the main contributions to anomalous vertical momentum transfers. The meridional scale of atmospheric motions is much larger than the width of the belts so that it makes sense to present hemispheric maps. We exclude, however, the tropical belt from the analysis because the pressure torques variances are so small there. As a basic hypothesis we assume that these regional contributions are similar to the time mean contributions in Fig. 3. On the other hand, torque events are short lived and must show a rapid evolution with increasing lag.

It is seen from Fig. 6a that the covariance pattern of PS has zonal wavenumber 4 but the maximum covariances are located outside the belt with downward transfers northwest and northeast of the Antarctic peninsula at $\tau=0$. These anomalies have small amplitudes at $\tau=-2$ days (not shown), then grow rapidly and can be recognized even at $\tau=2$ days but with their position shifted eastward (Fig. $6 \mathrm{~b})$. We have to conclude that pressure torque events in Antarctica are mainly due to synoptic systems at midlatitudes. This conclusion is supported by Fig. 6c, which demonstrates that the torque events in MS are similar to those in PS. The situation in Fig. $6 \mathrm{c}$ at $\tau=0$ and $\theta=300 \mathrm{~K}$ is quite similar to the mean in Fig. 3a so that indeed the 


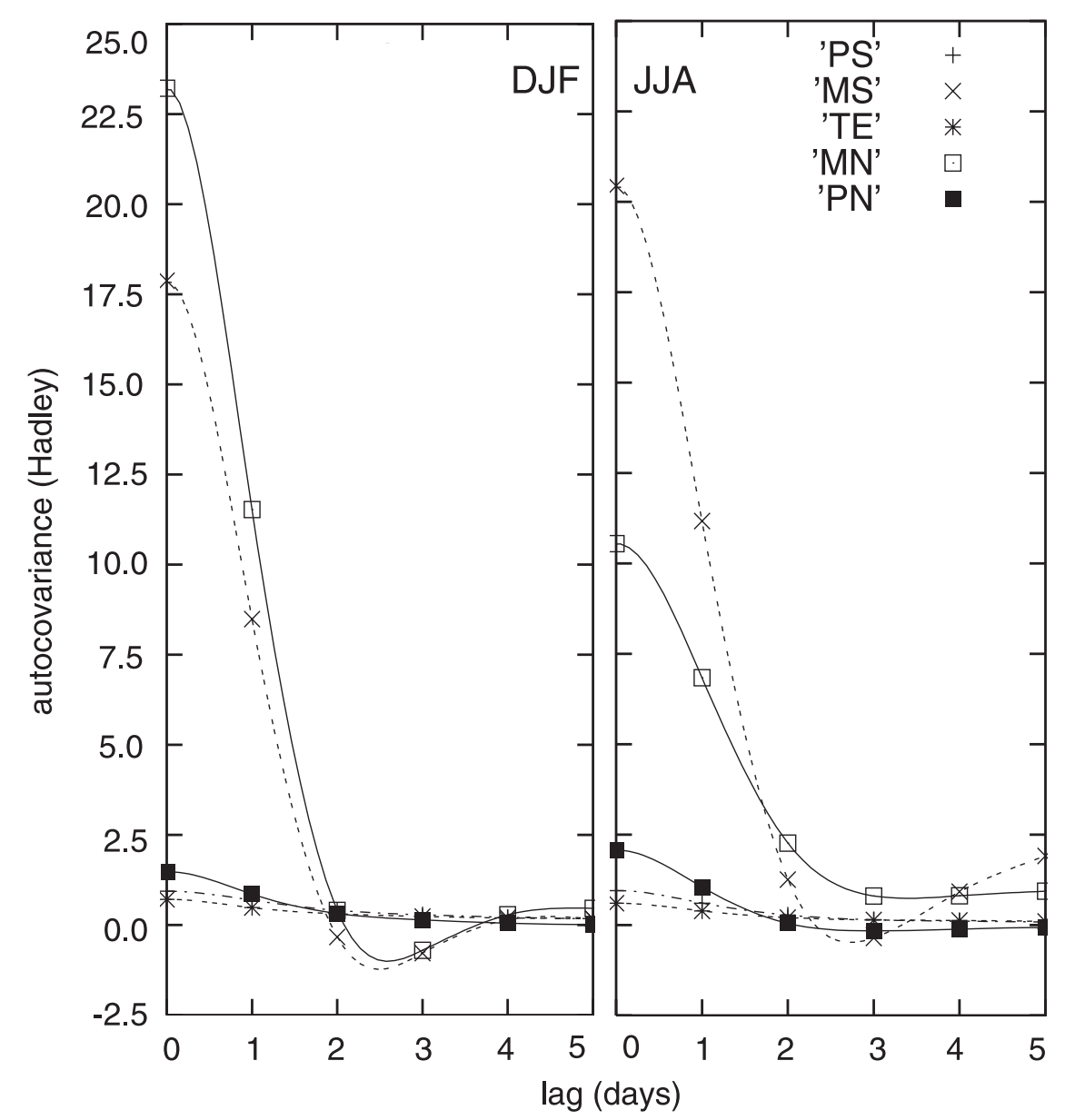

FIG. 5. Autocovariance of the pressure torques $T_{o}$ in Hadley for all belts $1-5$ as indicated and the $\theta$ surface $310 \mathrm{~K}$ as a function of lag (DJF and JJA).

anomalies appear to be enhanced mean distributions. However, 2 days later, it is the negative domains which dominate (Fig. 6d). This finding is also reflected in Fig. 5. Anomalies vanish within 2 days.

Similar to the southern polar belt (PS), the northern polar belt (PN) shows little variability within its own domain (not shown). The torque events in the northern midlatitude belt MN at $\tau=0$ (Fig. 6e) have a pattern that is reminiscent of the mean distribution except for the minimum in the northern Pacific in Fig. 3. However, the further evolution with increasing lag shows that anomalous torque contributions at and east of Rockies are negative and the area covered by these negative values extends almost to the Atlantic (Fig. 6f). Momentum is transferred upward above North America. These findings are broadly consistent with the intepretation of pressure torques in terms of baroclinic systems. The torques peak in the storm tracks and begin to disintegrate during their farther eastward motion. Momentum transfer may even be directed upward in this late stage.
It is also of interest to have a look at the height deviations related to the torques in a belt. The covariance $C\left(T_{o}, h \mid \tau\right)$ of the torque at $310 \mathrm{~K}$ in the belt MS (JJA) with the height field is displayed in Fig. 7 for the lags $\tau=0$ and $\tau=2$ days. At $\tau=0$, the height deviations are positive above South America and negative in the adjacent storm track regions. The scale of these height fields is much larger than that of synoptic systems. We have, however, to keep in mind that the fields in Fig. 7 are not individual height patterns. They represent kind of a height envelope of all perturbations related to anomalous torque events. Thus Fig. 7 indicates that the height of the $310-\mathrm{K}$ surface tends to be below normal at least in the mean during anomalous positive torque events. It is mainly warm low pressure systems that contribute to these events. Note that the height minima in Fig. 7 are located east of the maxima of $T_{\text {or }}$ in Fig. 6c. This suggests that much of the downward transfer occurs in the rear of the lows. This makes good sense. Johnson and Downey (1976) and Bates and Johnson (1984) demonstrated that 

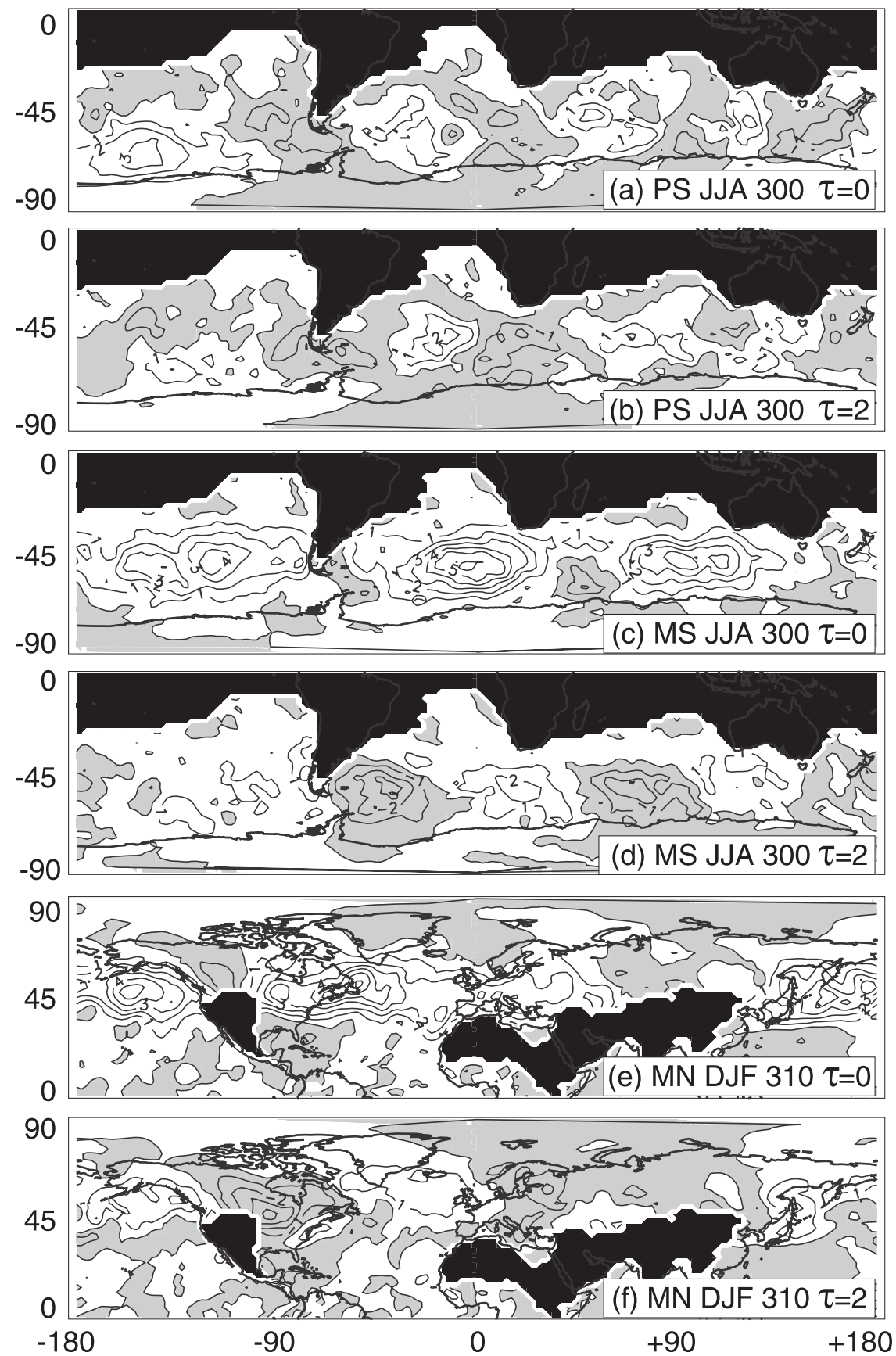

FIG. 6. Covariance of the normalized torque of various belts and the regional torque contributions $T_{\text {or }}$ (a) belt PS, JJA; $\theta=300 \mathrm{~K}, \tau=0$; (b) PS, JJA; $\theta=300 \mathrm{~K}, \tau=2$ days; (c) belt MS, JJA, $\theta=300 \mathrm{~K}, \tau=0$; (d) MS, JJA, $300 \mathrm{~K}, \tau=2$; (e) belt MN, DJF, $310 \mathrm{~K}, \tau=0$; and (f) MN, DJF, $310 \mathrm{~K}, \tau=2$ days. Shading as in Fig. 3; contour interval: 0.01 Hadley.

vertical AAM flux via pressure stresses is directed upward near the axis of active cyclones. Thus, the overall downward transfer must have its center west of the axis. The eastward propagation of the synoptic systems is seen clearly from Fig. 7b. It is however, rather surprising that there is an increase of the amplitudes. The synoptic systems appear to deepen on their way but their ability to exert torques is gone at $\tau=2$ days.

The situation in the northern belt is similar in many respects to that in MS. There is a ridge above North America and warm troughs in the storm track region. (Fig. 8). The minima in Fig. 8a tend to be located east of 

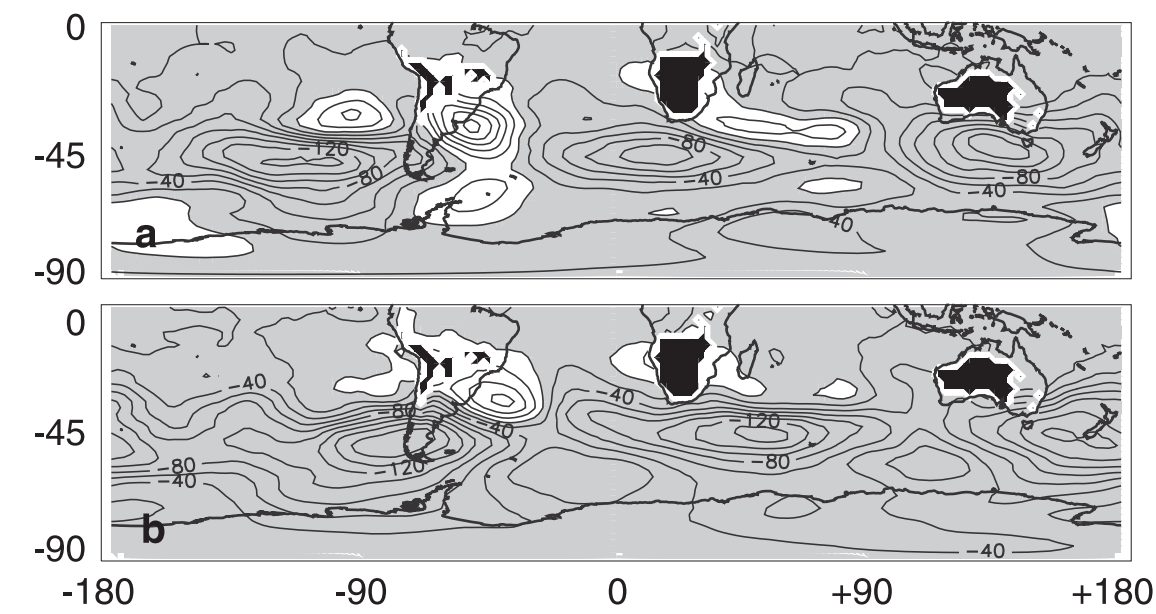

FIG. 7. Covariance of the normalized torque of belt MS with the height of the $310-\mathrm{K}$ surface in m; JJA: (a) $\tau=0$ and (b) $\tau=2$ days. Shading as in Figs. 3 and 6; contour interval: $20 \mathrm{~m}$.

the maxima in Fig. 6e. The ridge drifts eastward and so do the troughs. The Himalayan region shows a troughridge dipole at $\tau=0$. This structure is not obviously tied to an extremum of $T_{\text {or }}$. The "Himalayan minimum" merges with the trough approaching from the west.

\section{Discussion and conclusions}

The basic role of the pressure torques in isentropic coordinates is to bring AAM from the upper northward branch of the direct hemispheric cell down to the lower branch. The main results are presented in Figs. 4-8. They support the idea based on an analysis of baroclinic wave dynamics that pressure torques are strong and positive in the storm track regions. There is reasonably good qualitative agreement of pressure torque contributions and sensible heat transports as suggested by (1.12) for the zonal means. New and as yet unexplained features are the regions of upward transfer west of the American Cordillera, above Greenland, and west of southern Europe. There are also indications of leeside maxima. The Himalayas do not really fit into this pattern of orographic modification. There are no upstream minima for $\theta<320 \mathrm{~K}$ and there are no obvious leeside maxima. The vertical AAM transports due to transient motion in the $z$ system are weaker than the torques and the related patterns bear little resemblance. This is possible because the meridional AAM fluxes differ so strongly between both coordinate systems.

The height fields connected with the torque events must be seen as envelopes of the height derivations of all torque events. Heights tend to be low in the storm tracks

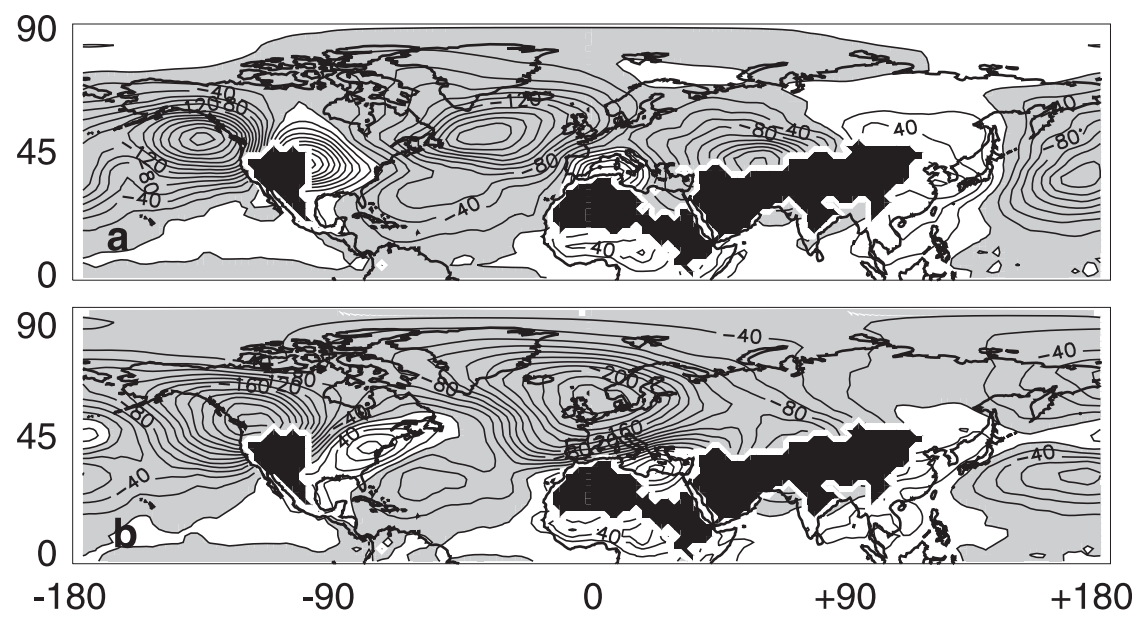

FIG. 8. Covariance of the normalized torque of belt $\mathrm{MN}$ with the height of the $310-\mathrm{K}$ surface in m; DJF: (a) $\tau=0$ and (b) $\tau=2$ days. Shading as in Fig. 3; contour interval: $20 \mathrm{~m}$. 
during anomalously strong downward torque events. These height deviations appear to deepen even after the torques reached their maximum intensity.

Statistical significance is a difficult but important issue. The ERA set provides a fairly large number of data per grid point. Thus, local standard significance tests give generally favorable results. There is, however, the wellknown counterargument that the values at neighboring grid points are statistically dependent so that a local test is not appropriate. One would have to project daily fields on a few basic patterns like EOFs and to perform the statistical test with respect to the corresponding principal components (e.g., Livezey and Chen 1983). The computational expense required was too large to perform such tests.

Altogether it is established by our results that the downward transfer of angular momentum at midlatitudes is concentrated in the western part of the Atlantic storm track and in the eastern part of the Pacific storm track in the Northern Hemisphere. There is little contribution by the torques above Asia. The situation is complex above North America. In the Southern Hemisphere, torque contributions are strong in the circumpolar storm track.

Acknowledgments. The authors thank Donald Johnson and two anonymous referees for their encouraging and helpful comments. Constructive remarks by K. Weickmann are also appreciated.

\section{REFERENCES}

Andrews, D., 1983: A finite-amplitude Eliassen-Palm theorem in isentropic coordinates. J. Atmos. Sci., 40, 1877-1883.

Bates, G., and D. Johnson, 1984: Mass and angular momentum diagnostics of cyclones with numerically simulated baroclinic waves. Mon. Wea. Rev., 112, 246-258.
Edmon, H., B. Hoskins, and M. McIntyre, 1980: Eliassen-Palm cross sections for the troposphere. J. Atmos. Sci., 37, 26002616.

Egger, J., and K.-P. Hoinka, 2008: The angular momentum budget of the TEM equations. J. Atmos. Sci., 65, 3305-3314.

$\longrightarrow$, and — 2009: Isentropic pressure and mountain torques. Mon. Wea. Rev., 137, 3047-3054.

—, K. Weickmann, and K.-P. Hoinka, 2007: Angular momentum in the global atmospheric circulation. Rev. Geophys., 45, RG4007, doi:10.1029/2006RG000213.

Holton, J., 1992: An Introduction to Dynamic Meteorology. Academic Press, 511 pp.

Hoskins, B., and K. Hodges, 2002: New perspectives on the Northern Hemisphere winter storm tracks. J. Atmos. Sci., 59, 1041-1061.

Johnson, D., 1980: A generalized transport equation for use with meteorological coordinate systems. Mon. Wea. Rev., 108, 733-744.

- 1989: The forcing and maintenance of global monsoon circulations: An isentropic analysis. Advances in Geophysics, Vol. 31, Academic Press, 63-329.

— budget equations for storms: Quasi-Lagrangian diagnostics I. Mon. Wea. Rev., 103, 967-979.

$\longrightarrow$, and - 1976: The absolute angular momentum budget of an extratropical cyclone: Quasi-Lagrangian diagnostics. Mon. Wea. Rev., 104, 3-14.

Juckes, M., I. James, and M. Blackburn, 1994: On the momentum budget of the southern extratropics. Quart. J. Roy. Meteor. Soc., 120, 1017-1044.

Livezey, L., and W. Chen, 1983: Statistical field significance and its determination by Monte Carlo techniques. Mon. Wea. Rev., 111, 46-59.

Oort, A., and J. Peixoto, 1983: Global angular momentum and energy balance requirements from observations. Advances in Geophysics, Vol. 25, Academic Press, 355-490.

Tanaka, D., T. Iwasaki, S. Uno, M. Ujije, and K. Miyazaki, 2004: Eliassen-Palm flux diagnosis based on isentropic representation. J. Atmos. Sci., 61, 2370-2383.

Townsend, R., and D. Johnson, 1985: A diagnostic study of the isentropic zonally averaged mass circulation during the GARP Global Experiment. J. Atmos. Sci., 42, 1565-1579. 